\title{
Aggregation and Dispersion of Small Hydrophobic Particles in Aqueous Electrolyte Solutions
}

\author{
Ronen Zangi and B. J. Berne* \\ Department of Chemistry and Center for Biomolecular Simulations, Columbia University, 3000 Broadway, \\ New York, New York 10027 \\ Received: July 14, 2006; In Final Form: September 5, 2006
}

\begin{abstract}
The effect of salts on the solvent-induced interactions between hydrophobic particles dispersed in explicit aqueous solution is investigated as a function of the salt's ionic charge density by molecular dynamics simulations. We demonstrate that aggregates of the hydrophobic particles can be formed or dissolved in response to changes in the charge density of the ions. Ions with high charge density increase the propensity of the hydrophobic particles to aggregate. This corresponds to stronger hydrophobic interactions and a decrease in the solubility (salting-out) of the hydrophobic particles. Ions with low charge density can either increase or decrease the propensity for aggregation depending on whether the concentration of the salt is low or high, respectively. At low concentrations of low charge density ions, the aggregate forms a "micelle-like" structure in which the ions are preferentially adsorbed at the surface of the aggregate. These "micelle-like" structures can be soluble in water so that the electrolyte can both increase the solubility and increase aggregation at the same time. We also find, that at the concentration of the hydrophobic particles studied $(\sim 0.75 \mathrm{~m})$, the aggregation process resembles a first-order transition in finite systems.
\end{abstract}

\section{Introduction}

Solvent-induced interactions between nonelectrolytes are greatly influenced by the type of salt dissolved in the aqueous solution. This has many implications in a wide range of biological, medical, and chemical phenomena. For example, the solubility of proteins (cf. Hofmeister series ${ }^{1}$ ), phase boundaries of micellar solutions, catalytic activity of enzymes, cloud points of nonionic surfactants, protein-DNA interactions, and polymer swelling are all sensitive to the kind of salt present in solution. ${ }^{2,3}$

Experimental measurements of the solubility of small nonpolar solutes (argon, nitrogen, oxygen, methane, etc.) indicate that, in most cases, the hydrophobic interaction is enhanced by the addition of salts. ${ }^{4-8}$ The degree of enhancement (which is different for different salts) is expressed by a decrease in the solubility, or "salting-out", of the hydrophobic particles in salt solutions relative to that in pure water. Consequently, ions can be ranked according to their ability to salt-out a certain type of solute. In general, salting-out increases as the charge density of the ions increases. ${ }^{5,9,10}$ This relationship becomes significant at moderate salt concentrations $(0.1-1 \mathrm{M})$ and is stronger for anions than for cations. For small nonpolar solutes, an increase of the solubility in the salt solution relative to that in pure water (salting-in) is observed only for very large ions. ${ }^{4,5,7,11}$ However, salting-in behavior is enhanced for larger and/or polar solutes. ${ }^{10,12-15}$

For more than a century many mechanisms have been proposed to explain the effect of electrolytes on the properties of nonelectrolytes in aqueous solution. The first explanation ascribed the effect to the ability of the salts to absorb water. ${ }^{1}$ In this picture salting-out can be explained by assuming that some of the water molecules are attached to the electrolyte ions and are thereby removed from their role as solvent molecules.

* Address correspondence to this author.
Other models related the size of volume contractions, which generally take place on mixing salt and water, to the degree of salting-out. ${ }^{10,16,17}$ In a different approach, Debye and McAulay ${ }^{18}$ represented the solvent and the nonelectrolyte solute as continuous media whose interactions with the ions are determined by the macroscopic dielectric constant. As a result of the electric field existing around the ions, the component in the solution of higher dielectric constant is pulled preferentially into the field and the one with lower dielectric constant is forced out. Since the dielectric constant of water is normally higher than that of other neutral media, there is an increase of the water molecules around the ions while the less polar neutral component tends to concentrate in the low-field regions of the ions. Thus, a reduction of the solubility of the nonelectrolyte is due to the increase of the nonelectrolyte-to-water ratio in those regions of the solution containing the nonelectrolyte. The success of all these models is limited to the prediction of the relative effectiveness of the ions as salting-out agents; however, they fail to account, even qualitatively, for a number of experimental observations, such as the salting-in behavior by salts that cause volume contraction when dissolved in water or the salting-in of solutes with dielectric constant lower than that of water. The major obstacle to theoretical work arises from a complex indirect influence of the salt on the solvent-mediated interaction between the nonelectrolytes. The complexity of the indirect induced interactions is made evident, for example, by the fact that the same electrolyte can cause salting-in for one solute but saltingout for another solute. ${ }^{10}$

Solute solubility is often linearly proportional to the electrolyte concentration and can be represented empirically by the Setschenow equation, ${ }^{19} \log \left(S_{0} / S\right)=K_{\mathrm{s}} C_{\mathrm{s}}$, where $S_{0}$ and $S$ are the solubilities of the nonelectrolyte molecule in pure water and in the ionic solution, respectively, $C_{\mathrm{s}}$ is the concentration of the electrolyte, and $K_{\mathrm{S}}$ is the salting (or Setschenow) coefficient. $K_{\mathrm{s}}$ is positive for salting-out electrolytes and negative for salting-

\subsection{1/jp064475+ CCC: $\$ 33.50$ (C) 2006 American Chemical Society} Published on Web 10/17/2006 
in electrolytes. The Setschenow equation holds in general up to relatively high concentrations $(1-4 \mathrm{M})$. However, for some salts at high concentrations the relation is far from linear. ${ }^{4,15,20}$ In some cases, it is found that the salting coefficient can change sign from negative to positive, i.e., the solubility passes through a minimum, with increasing the electrolyte or cosolute concentration. ${ }^{14,21,22}$

Previous computer simulations that investigated the effect of salt on hydrophobic particles were done by calculating radial distribution functions, ${ }^{23,24}$ relative hydration free energies (excess chemical potentials), ${ }^{25-28}$ and potentials of mean force. ${ }^{29}$ Values of the excess chemical potential were found, in most cases, to increase in the salt solution relative to pure water.

In this paper we perform Molecular Dynamics (MD) simulations to investigate the tendency of Lennard-Jones (LJ) particles to aggregate as a function of the ionic strength of the salt solution. We find that in salts with high charge density ions, the propensity for aggregation of the LJ particles increases as the ionic charges increase. Thus, increasing the ionic charge density strengthens the hydrophobic interaction and increases salting-out. The effect induced by low charge density ions depends on their concentration. When the concentration of the salt is high, low charge density ions weaken aggregation and are likely to increase the solubility of the LJ particles. However, at low salt (or cosolute) concentrations, low charge density ions increase the propensity of the hydrophobic particles to aggregate by preferentially binding to the surface of the aggregate. The structure of the aggregate formed resembles that of a micelle. Nevertheless, it is not clear whether the formation of the "micelle-like" structure will cause salting-in or salting-out. This behavior induced by low ionic charges is in agreement with experimental results on the hydrophobic self-association of tertbutyl alcohol in aqueous solution as a function of urea concentration. ${ }^{30}$ The distinction between high and low charge density ions is related to the strength of the ion-water interaction. High (low) charge density ions bind water more strongly (weakly) than water binds to itself in bulk water. ${ }^{31}$

\section{Methods}

The system modeled in this study consists of 18 nonpolar (hydrophobic) LJ particles, dispersed in aqueous salt solutions. The LJ parameters of the hydrophobic particles were taken to be $\sigma_{\mathrm{ljp}}=0.50 \mathrm{~nm}$ and $\epsilon_{\mathrm{ljp}}=1.00 \mathrm{~kJ} / \mathrm{mol}$. These values are larger and smaller, respectively, by approximately $30 \%$ than those of the methane molecule. The number of water molecules in the absence of salt was 1229. Salt was introduced by randomly replacing 48 water molecules by 24 cations and 24 anions. The LJ parameters of the ions were taken to be the same as those of the hydrophobic particles. To represent salts with different ionic strength, the magnitude of the cations and anions charges were varied, simultaneously, from 0.20 to $1.75 e$, so that the solutions were always electrically neutral. [In this study, the variation of the charge density of the ions was accomplished by changing the charge, which assumes noninteger values, at constant diameter of the ions. Of course, in nature the charge of the ions can only be a whole number while the diameter of the ions can vary in a more continuous way.] The masses of the ions and each of the solute particles were taken to be 40.0 amu. The salt concentrations for all solutions were fixed at 1.0 $m$, but in molarity varied in the range $0.90-1.0 \mathrm{M}$ due to variations in the volume of the solutions. In addition to the simulations at fixed salt concentration, where the charge density of the ions varied, simulations with varying salt concentrations at fixed cationic and anionic charges, of $|q|=0.50 e$, were also conducted with 18 and 27 LJ particles.
The MD computer simulations were preformed with the GROMACS package version 3.2.1. ${ }^{32}$ The time step used for integrating Newton's equations of motion was 0.002 ps. To maintain the system at a constant temperature of $300 \mathrm{~K}$, a Berendsen thermostat was applied, using a coupling time of 0.1 ps. ${ }^{33}$ The system was also coupled to a barostat with a pressure of 1.0 bar, a coupling time of $1.0 \mathrm{ps,} \mathrm{and} \mathrm{an} \mathrm{isothermal}$ compressibility of $1 \times 10^{-5} \mathrm{bar}^{-1} .33$ The evaluation of the electrostatic forces was performed by using the Particle-Mesh Ewald method (with grid spacing of $0.12 \mathrm{~nm}$ and quadratic interpolation). The evaluation of the LJ interactions was performed with a twin range cutoff of 1.0 and $1.4 \mathrm{~nm}$. Interactions within the shorter cutoff were updated every step while longer range interactions were updated every 5 steps. The LJ interactions between different particles were computed by the arithmetic combination rule. Water molecules were represented by the SPC/E model. ${ }^{34}$ Their bond distances and angle were constrained by using the SETTLE algorithm. ${ }^{35}$

The equilibration stage of all the simulations was for $6.0 \mathrm{~ns}$ and the data collection stage for $40.0 \mathrm{~ns}$. The pair potential of mean force of the hydrophobic particles, $w(r)$, was determined from their pair distribution function, $g(r)$, via the relationship $w(r)=-R T \ln [g(r)]$. As a measure for the degree of aggregation, the size of the clusters formed by the LJ particles was calculated. Two LJ particles were considered to belong to the same cluster if the distance between them was smaller than 0.80 $\mathrm{nm}$, which is the distance of the first minimum in their radial distribution function.

\section{Results and Discussion}

The pair correlation function, $g(r)$, of the $18 \mathrm{LJ}$ particles solvated in salt solutions, at a concentration of $1.0 \mathrm{~m}$, with ionic charges in the range $0.50 \leq|q| \leq 1.50$, as well as in pure water, is plotted in Figure 1a. The magnitude of the first peak of $g(r)$ is lowest when no ions are present in the solution. In this case, $g(r) \rightarrow 1$ for $r>1.1 \mathrm{~nm}$, indicating a random distribution of the hydrophobic particles at these interparticle distances. Random distribution of the hydrophobic particles at large distances is also observed for salt solutions with ionic charges of $|q|=0.75$ and $1.15 e$. However, as the charge density of the ions decreases below $|q|=0.75 e$ or increases above $|q|=$ $1.15 e$, the magnitude of the first peak increases dramatically and the values of $g(r)$ decrease (below the value of 1) as the interparticle distance increases. The corresponding pair potentials of mean force, $w(r)$, are shown in Figure 1b. Note that since the aggregate is finite and the simulation sample is small, the absolute value of $w(r)$ cannot be properly obtained. Therefore, the curves for $w(r)$ were shifted such that at the largest distance $(r=1.684 \mathrm{~nm})$ their values were zero. For pure water and for salt solutions with $|q|=0.75,1.15 e, g(r) \rightarrow 1$ and $w(r) \rightarrow 0$ at large distances. This indicates that the mean force acting between two LJ particles, in the presence of 16 other LJ particles, is zero at large distances, suggesting that the LJ particles are dispersed in these solutions. In contrast, $w(r)$ for $|q|=0.50,0.60,1.20$, and $1.50 e$ has a gradient at large values of $r$, indicating an attractive force that acts between two LJ particles (in the presence of 16 other particles). Such a force acts to bring all the $\mathrm{LJ}$ particles together to form a large cluster (aggregate). As the ionic charges decrease below $|q|=0.75 e$, or increase above $|q|=1.15 e$, the tendency of the particles to form a cluster increases as indicated by the magnitude of the gradient at large distances and by the depth of the first minimum in the curves of $w(r)$.

The tendency of the LJ particles to aggregate was also measured by their potential energy values. In Figure 2, the 

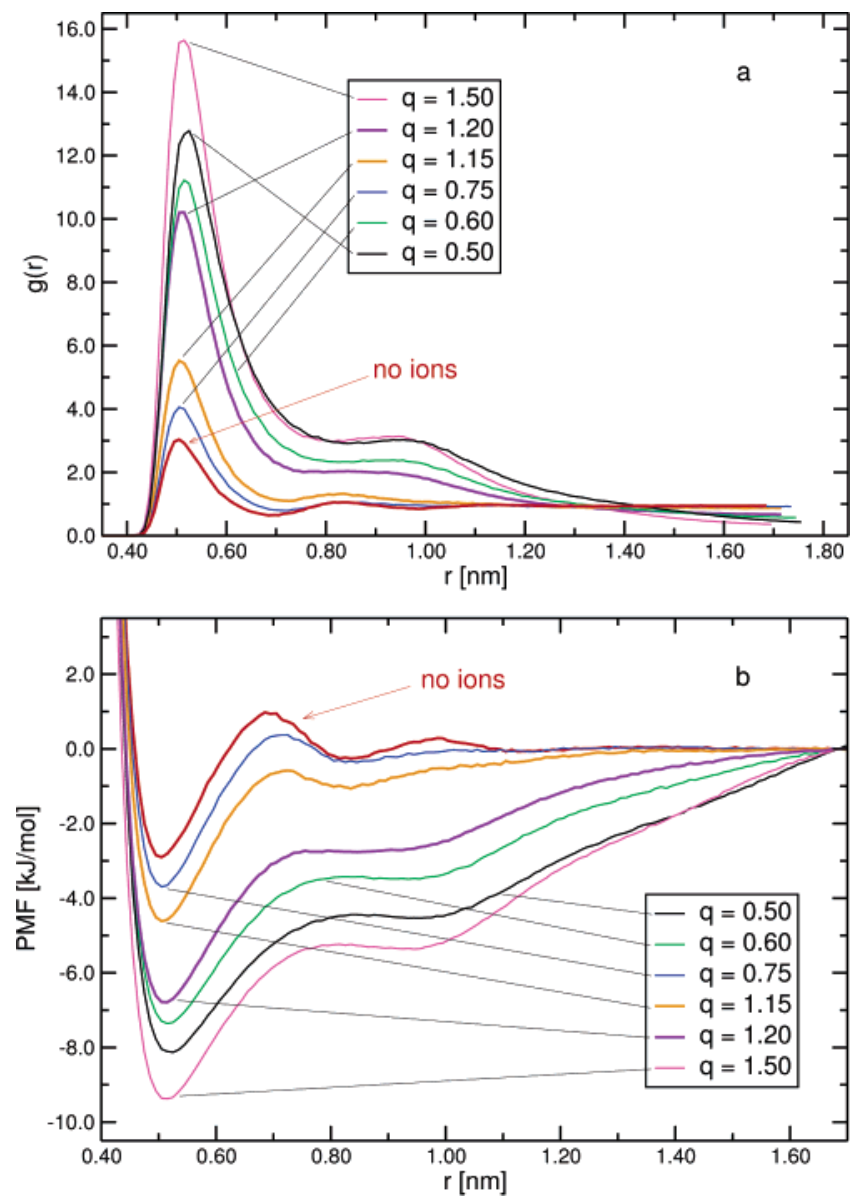

Figure 1. Results from simulations of $18 \mathrm{LJ}$ particles solvated in aqueous salt solutions at a concentration of $1.0 \mathrm{~m}$. The pair distribution function (a) and the two-body potential of mean force (b) of the LJ particles at several values of the ionic charge, $q$, as well as in pure water.

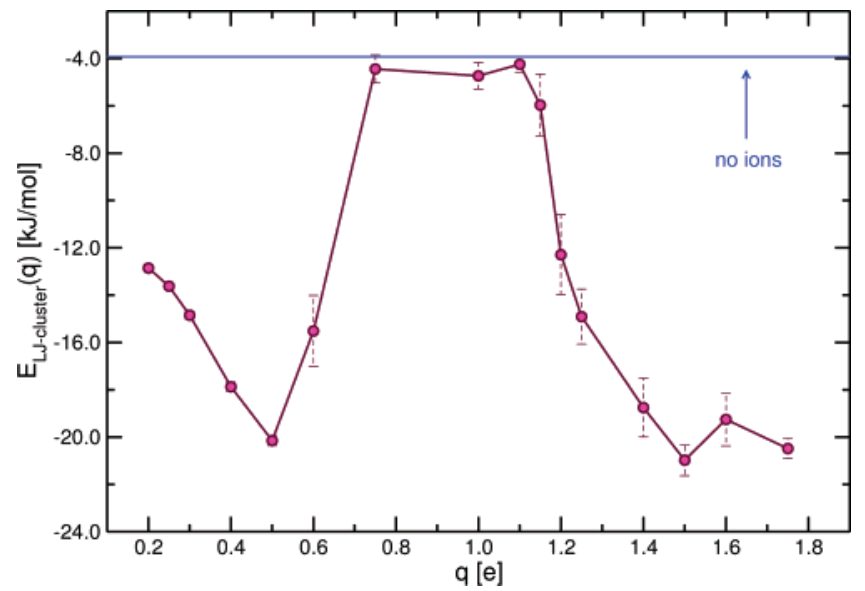

Figure 2. The potential energy of the $18 \mathrm{LJ}$ particles as a function of the ionic charge of the salt in the aqueous solution. The value obtained in pure water is indicated by the horizontal line. Negative values with large magnitude indicate a strong tendency for aggregation.

potential energy of the $18 \mathrm{LJ}$ particles as a function of the charges of the ions is plotted. Negative values with large magnitude indicate configurations where the particles are close to each other. The results inferred from Figure 2 are consistent with the results obtained from the calculations of $g(r)$ and $w(r)$ (displayed in Figure 1 for fewer salt solutions). The data show that the potential energy for all values of $q$ is lower than that of pure water, therefore, again indicating only an increase in the tendency for aggregation. For salts with charge densities in the range $1.10 \leq q \leq 1.50$, an increase in the propensity for aggregation as $q$ increases is observed. The increase in the tendency for aggregation observed in the simulation for this range of ionic charges is directly related to a decrease in the solubility (salting-out) of nonelectrolytes measured by experiments. In particular, lower solubilities are measured when the ionic strength of the solution increases. ${ }^{1,5,9,10}$ This behavior is also related to the trend observed in the ranking of the efficiency of ions to increase the stability of proteins native structure. ${ }^{2}$ It is likely that the mechanism of strengthening the hydrophobic interactions in this range of ionic charges is due to the fact that the ions are preferentially excluded from the vicinity of the hydrophobic particles and at the same time they are tightly bound to water (see Figure 4 for some physical evidence for this proposed explanation). Therefore, the number of water molecules available for solvating the hydrophobic particles decreases. Salt solutions with ionic charges $0.75 \leq|q|<1.10$ $e$ do not alter much the tendency of the LJ particles to aggregate relative to pure water. The strength of the interactions of these ions with water is similar to the strength of the water-water interactions. However, for lower charge density ions, $0.50 \leq$ $|q| \leq 0.75 e$, there is an increase in the propensity for aggregation with decreasing charge density of the ions (a behavior that was also shown in Figure 1). In this range of ionic charges there is a preferential adsorption of the ions on to the surface of the LJ aggregate (see below), forming a "micellelike" structure where the hydrophobic particles are buried inside. Depending on the concentrations of the hydrophobic particles and the ions, it is possible that several of these "micelle-like" aggregates will be dispersed in solution and stable with respect to the formation of an insoluble phase. In this case, the hydrophobic particles can exhibit an increased solubility relative to pure water (salting-in) because of "micellization". On the other hand, if the concentration of the ions (cosolutes) is small enough relative to the concentration needed to stabilize the surfaces of these aggregates, but still large enough to stabilize the interface that forms upon phase separation, it is possible that the solubility of the hydrophobic particles (relative to pure water) will decrease (salting-out). In both cases, the low charge density ions act as "surfactants" by reducing the surface tension of the interface between the aqueous solution and the hydrophobic particles. From the series of simulations performed in this study, it is not possible to determine the trend in the solubility of the hydrophobic particles when they aggregate with the formation of a "micelle-like" structure. Note that the low charge density ions in this study have fractional elementary charge and form a diffusive double layer around the aggregate. Therefore, the effect induced by these ions may be similar to the effect caused by neutral polar molecules that are added as cosolute to the aqueous solution. As the ionic charge decreases further, $0.20 \leq|q|<0.50 e$, there is a decrease in the attractions between the LJ particles. This is because the ions become so similar to the LJ particles that they penetrate the insoluble cluster (breaking the "micelle-like" structure) rendering the interparticle distances between the nonelectrolyte solute larger. By inspecting instantanous configurations we observed that the trajectory for $|q|=0.40 e$ contained cases where the "micelle-like" aggregate extended throughout the simulation box due to the application of periodic boundary conditions. ${ }^{36,37}$ The number of these cases increases as $|q|$ decreases.

Note that the propensity for aggregation decreases with increasing ionic charge in the range $1.50 \leq q \leq 1.75 e$. This is due to a transition in the distribution of the ions that occurs for 


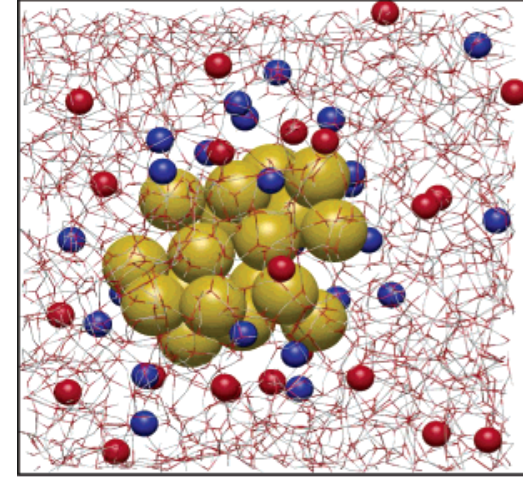

$q=0.50$

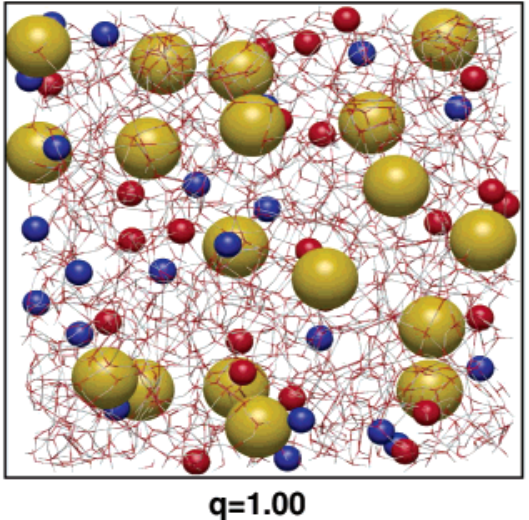

$\mathrm{q}=\mathbf{1 . 0 0}$

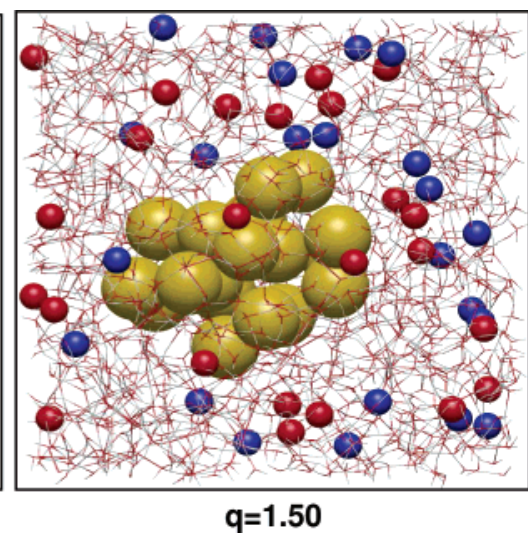

$\mathrm{q}=\mathbf{1 . 5 0}$

Figure 3. Snapshots of the simulation box showing the configuration of the $18 \mathrm{LJ}$ particles in the presence of salts with different ionic charges. The LJ particles are depicted in yellow, positive ions in red, and negative ions in blue. The hydrogen bond network of the water molecules is shown by thin lines. The sizes of the different atoms are not plotted according to their excluded volume.

ions with high charge density. In this case, the ions form heterogeneous regions of high and low salt concentration, a phenomenon that has been observed in computer simulations of aqueous solution of ammonium sulfate ${ }^{26}$ and in NMR experiments. ${ }^{38}$

Instantaneous configurations of the simulation box for salts with $|q|=0.50,1.00$, and $1.50 e$ are displayed in Figure 3. The high propensity for aggregation of the LJ particles when ions with $|q|=0.50$ or $1.50 e$ are present in solution is clearly evident by the large LJ cluster formed, while ions with $|q|=1.00 e$ yield a solution where the LJ particles are highly dispersed, as they are when no ions are present in the solution (no figure is shown for this latter case). Ions with $|q|=0.50 e$ are attracted to the cluster while the ions with $|q|=1.50 e$ are depleted from it. This is better seen in Figure 4 where the pair correlation functions between the hydrophobic particles and the cations as well as with the anions are plotted. Strong adsorption and strong depletion of the ions from the surface of the hydrophobic cluster is evident for ionic charge of $|q|=0.50$ and $1.50 e$, respectively. For the former case there is an excess of negative charge at the aggregate-solution interface, while for the latter case there is a slight excess of positive charge at the aggregate solution interface. Adsorption of negative ions at the water-hydrophobic interface is observed experimentally by measurements of the surface potential at the air-water interface of aqueous solution of electrolytes with comparable size of anions and cations. ${ }^{39}$ The negative surface potential at the air-water interface is due to the asymmetry of the charge distribution (quadrupole moment) of the water molecule. ${ }^{40}$ Note that from a thermodynamic point of view, the change of the chemical potential of a dissolved macromolecule upon the addition of cosolute to the solution is related to the preferential binding of the cosolute to the macromolecule. ${ }^{41-44}$ It is likely that the chemical potential of the aggregate is related to preferential binding or exclusion of the ions. Nevertheless, the propensity of the LJ particles to aggregate is clearly not a simple function of preferential binding or exclusion.

The aggregate is not static and is in equilibrium with other aggregates of different sizes. In Figure 5 the probabilities (normalized to one) of observing clusters with different sizes are plotted for few salt solutions and for pure water. For $|q|=$ $1.00 e$ and for pure water the majority of the clusters consist of either 1 or 2 particles and there is a rapid drop off of the probabilities with increasing cluster size. On the other hand, for ions with $|q|=0.50$ and $1.50 e$ the majority of the clusters are of size in the range of $16-18$ particles. There is also a small
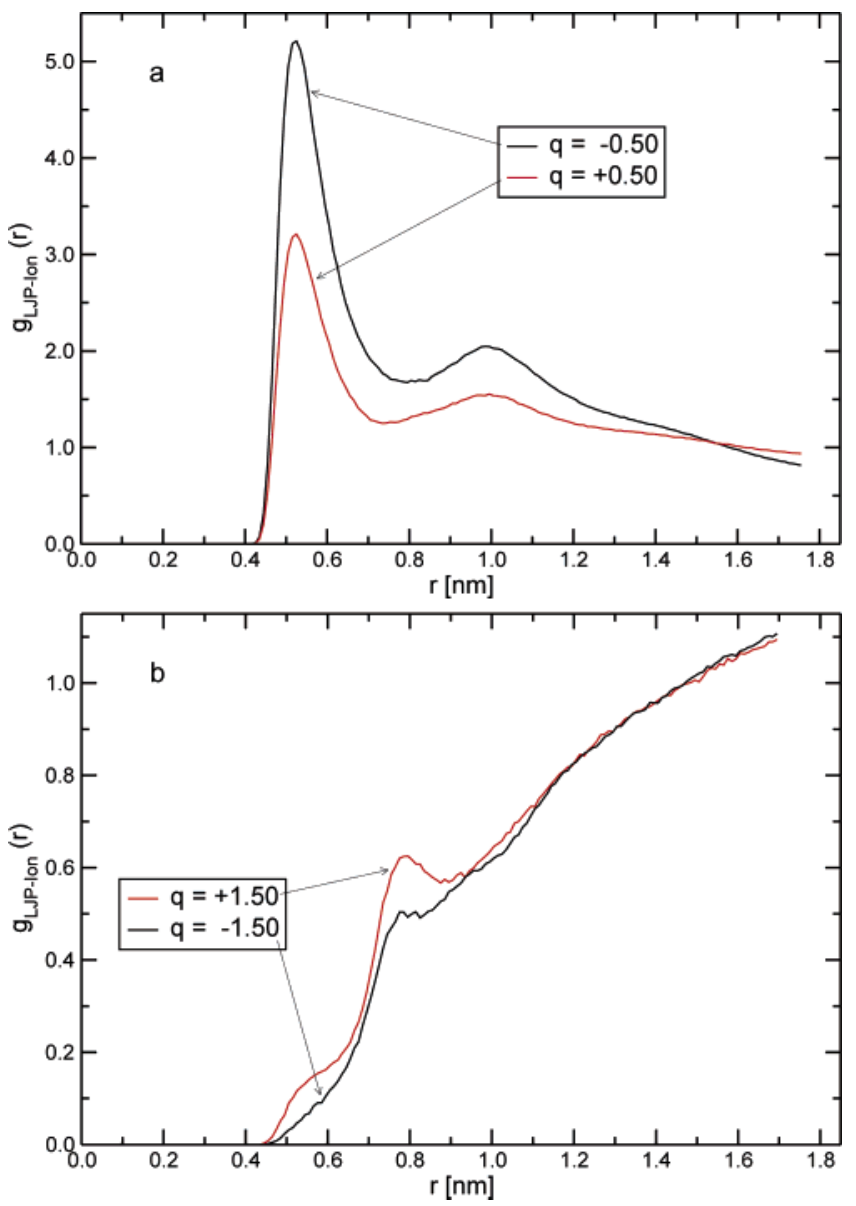

Figure 4. Radial distribution functions between the LJ particles and the cations as well as with the anions for salt solutions with ionic charge: (a) $|q|=0.50 e$ and (b) $|q|=1.50 e$.

probability to find clusters of 1 or 2 particles. The transition region $(|q|=0.60$ and $1.25 e)$ from solutions where the $\mathrm{LJ}$ particles are highly dispersed to solutions where they form large aggregates is characterized by a bimodal distribution where the probability of clusters with intermediate size is very small, suggesting that the aggregation process induced in this finite system has the characteristics of an incipient first-order phase transition. During the time period of the simulations (40 ns) we observe 4-9 transitions between the aggregated state (cluster with 16-18 particles) and the dispersed state (cluster(s) with maximum size of $2-3$ particles) for salt solutions with charge densities around the transition points. 

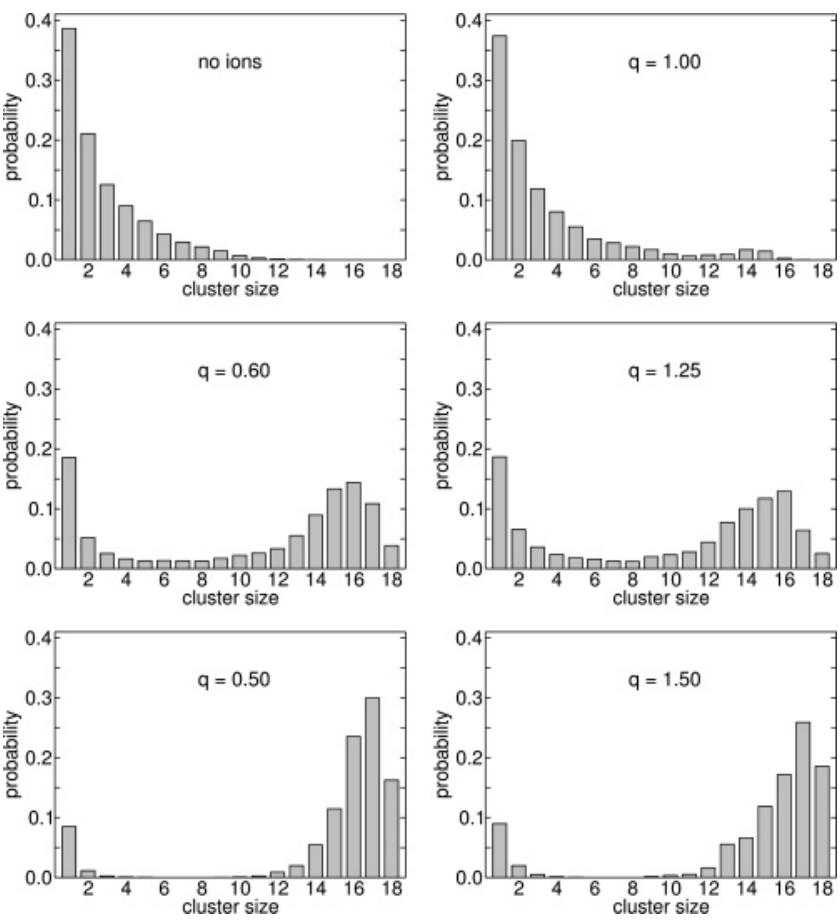

Figure 5. Probability histograms (normalized to one) of finding a cluster of the hydrophobic particles as a function of its size in the presence of ions with different charge densities as well as in pure water.

NMR studies of the effect of urea on the hydrophobic selfassociation of tert-butyl alcohol in aqueous solutions showed that low urea concentrations enhance the hydrophobic selfassociation. However, at higher urea concentrations a destabilization of the hydrophobic interaction is observed. ${ }^{30}$ With low tert-butyl alcohol (solute) concentrations this enhancement effect reached a pronounced maximum. No explanation has been offered to account for these results. As we noted before, polar neutral molecules that are added as cosolute to the solution may behave similarly to our model for salts with low ionic charges. We performed additional simulations to investigate the selfassociation of the hydrophobic particles in salt solution with ionic charge of $|q|=0.50 \mathrm{e}$. Two separate series of simulations with 18 and $27 \mathrm{LJ}$ particles were performed, corresponding respectively to dispersed and aggregated assembly of particles in pure water. Figure 6 displays the potential energy (per particle) of the hydrophobic particles (a measure to their tendency to self-associate) as a function of the salt concentration. In both cases, a maximum in the propensity to self-associate is observed as the concentration of the salt (cosolute) increases. At low cosolute concentration there is an increase in the tendency for aggregation since these ions of low charge are particles with hydrophobic character, their presence in solution acts as if the concentration of the solute (hydrophobic particles and low charge ions) increases. The increase in the concentration of "hydrophobic particles" should allow them to nucleate and to form stable aggregate or phase separate. As observed in the experiment, ${ }^{30}$ the enhancement of the tendency for aggregation, by the hydrophobic interactions, is stronger for the lower concentration of the hydrophobic particles. With further increase in the cosolute (salt) concentration a decrease in the propensity of the hydrophobic particles to aggregate is observed and is probably due to a decrease in the dielectric properties of the electrolyte-water matrix so the hydrophobic particles are more soluble in this medium. This occurs when the amount of the cosolute is large enough to act as a cosolvent in which the solute can be dissolved. The situation we observed for very low charge
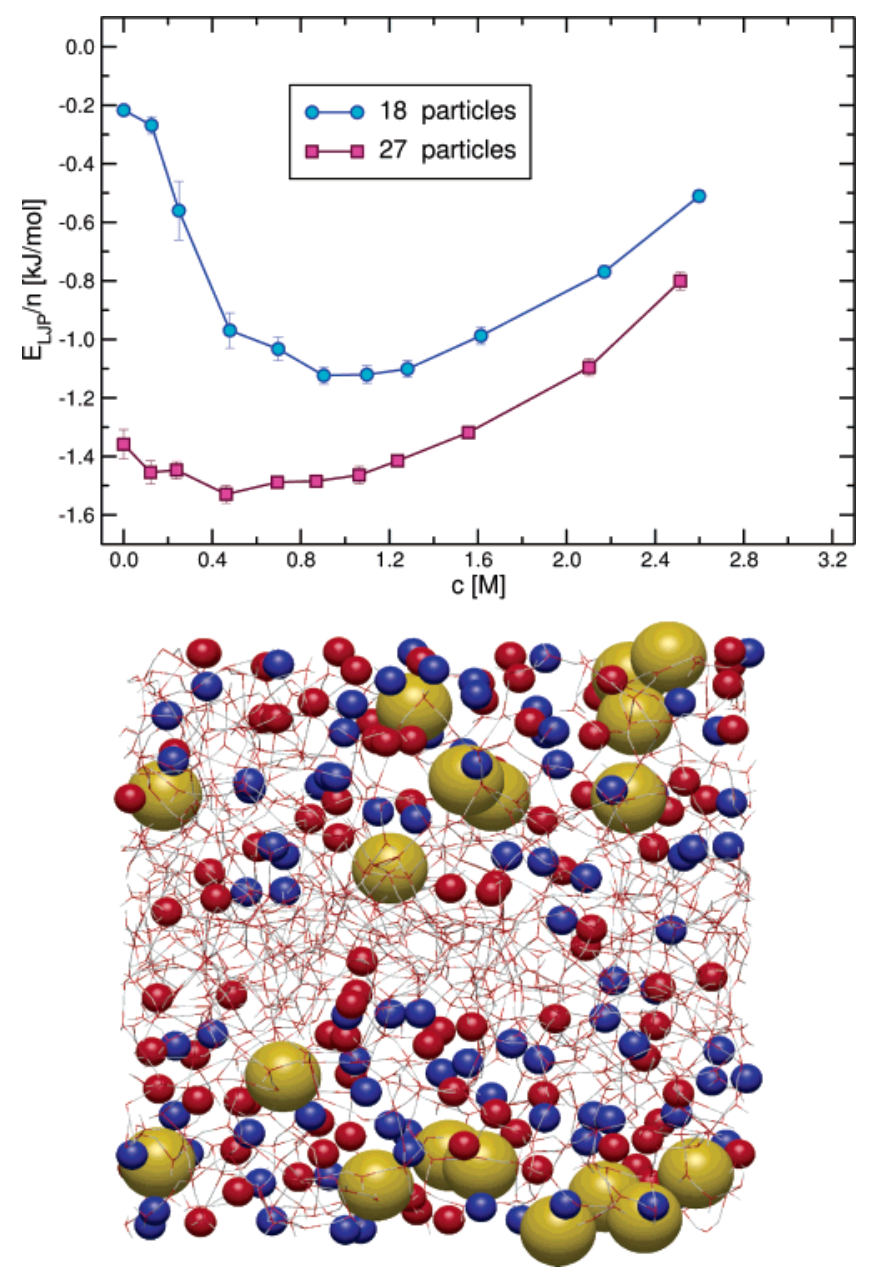

Figure 6. (Top) The potential energy (per particle) of the hydrophobic particles (a measure for the propensity for self-association) as a function of the salt concentration for $q=0.50 e$ plotted for simulations with 18 and $27 \mathrm{LJ}$ particles. (Bottom) Snapshot of the simulation box for $|q|=0.50 e$ with $18 \mathrm{LJ}$ particles at a salt concentration of $2.60 \mathrm{M}$ (the color code is the same as in Figure 3).

density ions $0.20 \leq q \leq 0.40$ we also observe for very high concentrations (for the $18 \mathrm{LJ}$ particles system above a salt concentration of $2.1 \mathrm{M}$ and for the $27 \mathrm{LJ}$ particle system above a salt concentration of $1.5 \mathrm{M}$ ). For these high concentrations, the complex of LJ particle and ions can extend over the entire simulation box due to the periodic boundary condition with irregular shapes of cylinders or sheets. ${ }^{36,37}$ Figure 6 displays an example of such a configuration.

The solubility of hydrophobic particles in aqueous solution as a function of cosolute concentration with weaker interaction to water than the water-water interaction can be complex. This is seen in experimental measurements of the solubility of argon in an aqueous solution of ethanol, ${ }^{21}$ tert-butyl alcohol, ${ }^{22} p$ dioxane, ${ }^{45}$ ethyleneglycol, ${ }^{46}$ and glycine in an aqueous solution of alkali bromide salts. ${ }^{14}$ In these studies, the observed solubility as a function of the cosolute (electrolyte) mole fraction displayed a minimum, and in some cases an additional maximum, at low cosolute concentrations. Although our simulation results cannot be directly compared to these experimental studies, they can provide qualitative explanations for the complex solubility measurements observed for small hydrophobic particles.

Micelles, vesicles, and bilayers are built by amphiphilic molecules with polar headgroups and hydrophobic tails (e.g., phospholipids). The function of the headgroup is to stabilize the structure in which there is aggregation of the hydrophobic 
segments. The results obtained from our simulations with low charge density ions (where, for example, the association of the LJ particles is stronger in a salt solution with $|q|=0.5$ than 0.6) suggest that, in addition, the magnitude of the hydrophobic interactions between the tails is sensitive to the charge density of the headgroup of the amphiphile. This raises an intriguing question of whether this effect plays a role in the variations in the chain-melting temperature of lipid bilayers with different headgroups measured experimentally. ${ }^{47}$ The stronger the hydration free energy of the headgroup the lower the chain-melting temperature of the membrane.

\section{Conclusions}

In this work we studied the effect of the charge density of ions in aqueous salt solution on the propensity of hydrophobic particles to aggregate. A systematic variation in the charge density of the ions was performed by changing the charge at constant ionic diameter. The choice of taking equal anionic and cationic diameters restricts the maximum and minimum salt ionic charges we could consider. If the charge is too large or too small the ions are not dispersed in solution and form aggregates. This effect is evident experimentally by measurements of the solubility of simple inorganic salts. Large cations with small anions, or vice versa, give highly soluble salts whereas salts containing cations with size comparable to the size of the anions will tend to pair (because they interact strongly with each other) and form salts with moderate to low solubility. ${ }^{48}$

Although the number of variables we changed in this study was limited, we demonstrated that the ionic strength of aqueous solutions strongly affects the magnitude of the hydrophobic interaction. This was observed by changes in the propensity of the hydrophobic particles to aggregate or disperse in different salt solutions. We made the following observations:

Salts with sufficiently high charge density ions increase the propensity for the hydrophobic particles to aggregate, and this propensity increases with ionic charge density.

Salts with low charge density ions also can increase the tendency for hydrophobic aggregation. In this case, the ions are preferentially bound to the surface of the cluster of hydrophobic particles forming a "micelle-like" structure. This aggregate is stable at low salt (cosolute) concentrations. However, with increasing salt (cosolute) concentration the hydrophobic particles become disspersed rather than aggregated.

The propensity of the hydrophobic particles to aggregate is clearly not a monotonic function of the preferential binding ${ }^{42-44}$ (or exclusion) of the ions from the hydrophobic particles, as has been suggested in the literature. ${ }^{41-44}$

Acknowledgment. This research was supported by the National Science Foundation via grant no. NSF-CHE-03-16896.

\section{References and Notes}

(1) Hofmeister, F. Arch. Exp. Pathol. Pharmakol. 1888, 25, 1-30.

(2) Collins, K. D.; Washabaugh, M. W. Q. Rev. Biophys. 1985, 18, $323-422$.
(3) Cacace, M. G.; Landau, E. M.; Ramsden, J. Q. Rev. Biophys. 1997, 30, 241-277.

(4) Randall, M.; Failey, C. F. Chem. Rev. 1927, 4, 271-284. 1777.

(5) McDevit, W. F.; Long, F. A. J. Am. Chem. Soc. 1952, 74, 17731466.

(6) O’Sullivan, T. D.; Smith, N. O. J. Phys. Chem. 1970, 74, 1460-

(7) Ben-Naim, A.; Yaacobi, M. J. Phys. Chem. 1974, 78, 170-175.

(8) Leshchev, S. M.; Novik, N. P. J. Struct. Chem. 2004, 45, 323326.

(9) Albright, P. S. J. Am. Chem. Soc. 1937, 59, 2098-2104.

(10) Long, F. A.; McDevit, W. F. Chem. Rev. 1952, 51, 119-169.

(11) Feillolay, A.; Lucas, M. J. Phys. Chem. 1972, 76, 3068-3072.

(12) Neuberg, C. Biochem. Z. 1916, 76, 107-176.

(13) Gross, P. M. Chem. Rev. 1933, 13, 91-101.

(14) Meyer, K. H.; Klemm, O. Helv. Chim. Acta 1940, 23, 25-27.

(15) Robinson, D. R.; Grant, M. E. J. Biol. Chem. 1966, 241, 40304042.

(16) Euler, H. Z. Phys. Chem. 1899, 31, 360-369.

(17) Gibson, R. E. J. Am. Chem. Soc. 1934, 56, 4-14.

(18) Debye, P.; McAulay, J. Phys. Z. 1925, 26, 22-29.

(19) Setschenow, J. Z. Phys. Chem. 1889, 4, 117-125.

(20) Ruetschi, P.; Amlie, R. F. J. Phys. Chem. 1966, 70, 718-723.

(21) Ben-Naim, A.; Baer, S. Trans. Faraday Soc. 1964, 60, 1736-1741.

(22) Cargill, R. W.; Morrison, T. J. J. Chem. Soc., Faraday Trans. 1

$1975,71,618-624$.

(23) Mancera, R. L. Chem. Phys. Lett. 1998, 296, 459-465.

(24) Paschek, D.; Geiger, A.; Hervé, M. J.; Suter, D. J. Chem. Phys. 2006, 124, 154508 .

(25) Hummer, G.; Garde, S.; Garcia, A. E.; Paulaitis, M. E.; Pratt, L. R. J. Phys. Chem. B 1998, 102, 10469-10482.

(26) Smith, P. E. J. Phys. Chem. B 1999, 103, 525-534.

(27) Kalra, A.; Tugcu, N.; Cramer, S. M.; Garde, S. J. Phys. Chem. B 2001, 105, 6380-6386.

(28) Hribar, B.; Southall, N. T.; Vlachy, V.; Dill, K. A. J. Am. Chem. Soc. 2002, 124, 12302-12311.

(29) Ghosh, T.; Kalra, A.; Garde, S. J. Phys. Chem. B 2005, 109, 642651.

(30) Mayele, M.; Holz, M. Phys. Chem. Chem. Phys. 2000, 2, 24292434.

(31) Collins, K. D. Proc. Natl. Acad. Sci. U.S.A. 1995, 92, 5553-5557.

(32) Lindahl, E.; Hess, B.; van der Spoel, D. J. Mol. Mod. 2001, 7, 306-317.

(33) Berendsen, H. J. C.; Postma, J. P. M.; van Gunsteren, W. F.; DiNola, A.; Haak, J. R. J. Chem. Phys. 1984, 81, 3684-3690.

(34) Berendsen, H. J. C.; Grigera, J. R.; Straatsma, T. P. J. Phys. Chem. 1987, 91, 6269-6271.

(35) Miyamoto, S.; Kollman, P. A. J. Comput. Chem. 1992, 13, 952962.

(36) Wallqvist, A. J. Phys. Chem. 1991, 95, 8921-8927.

(37) Raschke, T. M.; Tsai, J.; Levitt, M. Proc. Natl. Acad. Sci. U.S.A. 2001, 98, 5965-5969.

(38) Dillon, S. R.; Dougherty, R. C. J. Phys. Chem. A 2003, 107, 1021710220

(39) Jarvis, N. L.; Scheiman, M. A. J. Phys. Chem. 1968, 72, 74-78.

(40) Frenkel, J. Kinetic Theory of Liquids; Oxford University Press: Oxford, UK, 1946

(41) Tanford, C. J. Mol. Biol. 1969, 39, 539-544.

(42) Arakawa, T.; Timasheff, S. N. Biochemistry 1984, 23, 5912-5923.

(43) Schellman, J. A. Biopolymers 1987, 26, 549-559.

(44) Parsegian, V. A.; Rand, R. P.; Rau, D. C. Proc. Natl. Acad. Sci. U.S.A. 2000, 97, 3987-3992.

(45) Ben-Naim, A.; Moran, G. Trans. Faraday Soc. 1965, 61, 821825.

(46) Ben-Naim, A. J. Phys. Chem. 1968, 72, 2998-3001.

(47) Cevc, G.; Seddom, J. M.; Marsh, D. Faraday Discuss. Chem. Soc. 1986, 81, 179-189.

(48) Collins, K. D. Biophys. J. 1997, 72, 65-76. 